\title{
Estrutura populacional de Callinectes ornatus Ordway (Crustacea, Portunidae) no Balneário Shangri-Lá, Pontal do Paraná, Paraná, Brasil ${ }^{1}$
}

\author{
Cassiana Baptista ${ }^{2}$, Marcelo A. Amaro Pinheiro ${ }^{3}$, Arno Blankensteyn ${ }^{4} \&$ Carlos A. Borzone ${ }^{5}$ \\ ${ }^{1}$ Contribuição número 1459 do Departamento de Zoologia, Universidade Federal do Paraná. \\ 2 Departamento de Zoologia, Universidade Federal do Paraná. Caixa Postal 19020, 81531-980 Curitiba, Paraná, Brasil. \\ E-mail: cassian@bio.ufpr.br \\ ${ }^{3}$ Grupo de Estudos em Biologia de Crustáceos, Universidade Estadual Paulista. Praça Infante Dom Henrique, 11330 -900 São \\ Vicente, São Paulo, Brasil. \\ ${ }^{4}$ Centro de Ciências Biológicas, Departamento de Ecologia e Zoologia, Universidade Federal de Santa Catarina. Caixa Postal \\ 47, 688010-970 Florianópolis, Santa Catarina, Brasil. \\ ${ }_{5}^{5}$ Centro de Estudos do Mar, Universidade Federal do Paraná. Avenida Beira Mar, 83255-000 Pontal do Paraná, Paraná, \\ Brasil.
}

\begin{abstract}
Population structure of $C$. ornatus Ordway (Crustacea, Portunidae) in Shangri-lá Beach, Pontal do Paraná, Paraná, Brazil. Swimming crabs are commonly found in the otter-trawl shrimp fishery by-catch, which operate intensively in the Coastal Plain of Leste Beach, where is located Shangri-lá Beach $\left(25^{\circ} 37^{\prime} 30^{\prime \prime} \mathrm{S}\right.$ and $48^{\circ} 25^{\prime} 08^{\prime \prime}$ W), Paraná State. This work aimed to study the population structure of Callinectes ornatus Ordway, 1863, the most abundant swimming crab in this beach. Between March 2000 and April 2001, fortnightly visits were accomplished in Shangri-lá at the fish market, where all swimming crabs of one otter-trawl were collected. The following data from each individual of $C$. ornatus were obtained: sex, carapace width (excluding lateral spines), weight, and the gonadal maturation stage, based on its color and size. Juveniles and mature males were dominant, mean sexual proportion was 2.2:1 (males:females). Two recruitment periods were detected, but few ovigerous females were obtained. Size at sexual maturation was estimated at $55 \mathrm{~mm}$ for males, and $48 \mathrm{~mm}$ for females. Both sexes presented positives allometric growths. The results indicate that an important portion of $C$. ornatus population is captured in the otter-trawl shrimp fishery in Shangri-lá Beach (Paraná). Such information is important for futures plans of by-catch management in that area.
\end{abstract}

KEY WORDS. By-catch, maturity, population biology, sex ratio, swimming crab.

Os portunídeos distribuem-se ao longo da costa Atlântica Ocidental, desde a América do Norte até o extremo sul da América do Sul (Fao 1993, Melo 1996). Muitas espécies sofrem exploração econômica intensa, como é o caso do siri azul Callinectes sapidus Rathbun, 1896, um dos principais recursos pesqueiros do leste e sudeste dos EUA, tendo sido intensamente estudado (Williams 1974, Lipcius \& VAN Engel 1990, Jordan 1998).

Nas regiões sudeste e sul do Brasil, os portunídeos que se têm destacado por sua abundância relativa, são Callinectes sapidus, Callinectes danae Smith, 1869 e Callinectes ornatus Ordway, 1863, sendo a primeira espécie encontrada preferencialmente em áreas estuarinas (BRANCO \& LUNARDON-Branco 1993a,b, Mantelatto \& Fransozo 1996, 1997, 1998, NegreirosFransozo et al. 1999). C. ornatus ocorre em praticamente todo o Atlântico Ocidental, da Carolina do Norte (EUA) ao Rio Grande do Sul (Brasil). Habita fundos de areia, lama ou conchas, com registros na região do entre-marés até 75 metros de pro- fundidade, principalmente na proximidade de rios e baías, em águas de salinidade moderada (MeLo 1999).

No Brasil, o conhecimento da biologia de C. ornatus tem recebido várias contribuições, destacando-se no Paraná os estudos realizados por BRANCO \& LunARDON-BranCo (1993 a,b), envolvendo a estrutura populacional, o crescimento e o tamanho de primeira maturação. No litoral norte de São Paulo, também foram analisadas a biologia populacional e a reprodução dessa espécie, com determinação do tamanho na primeira maturação e fecundidade (MANTELATTO \& Fransozo 1996, 1997), desenvolvimento gonadal e ciclo de muda (Mantelatto \& Fransozo 1998), estrutura populacional, razão sexual e outros aspectos reprodutivos (Negreiros-Fransozo et al. 1999). Outra importante fonte de informações sobre a reprodução de $C$. ornatus é o trabalho de HAEFNER (1990), enfocando a morfometria e tamanho de primeira maturação na Baía de Mullet, Bermudas.

Revista Brasileira de Zoologia 20 (4): 661-666, dezembro 2003 
Na costa paranaense, C. ornatus ocorre em grande quantidade como fauna acompanhante na pesca do camarão setebarbas, Xiphopenaeus kroyeri (Heller, 1862). Apesar da sua abundância, ele não é aproveitado comercialmente e geralmente passa a formar parte do rejeito dessa pescaria. O presente trabalho foi desenvolvido com o objetivo de prover informações sobre a biologia e a estrutura populacional de $C$. ornatus na costa paranaense, visando melhores condições de manejo.

\section{MATERIAL E MÉTODOS}

De abril/2000 a abril/2001, foram realizadas coletas quinzenais junto às bancas dos pescadores do Balneário Shangri-lá $\left(25^{\circ} 37^{\prime} 30^{\prime \prime}\right.$ e e $\left.48^{\circ} 25^{\prime} 08^{\prime \prime} \mathrm{W}\right)$, Pontal do Paraná, Paraná, Brasil. Cada amostra consistiu na coleta de todos os siris de pelo menos um arrasto (com duração aproximada de uma hora) que não tivesse a sua fauna acompanhante rejeitada ou selecionada. Os siris obtidos foram acondicionados em sacos plásticos e mantidos sob congelamento até seu manuseio.

Em laboratório, após a identificação e determinação do sexo de cada indivíduo (Melo 1996, Williams 1984), foi tomada a medida da largura da carapaça (entre a base dos espinhos laterais) com um paquímetro manual e o peso com uma balança digital. Os animais foram classificados segundo o estágio de desenvolvimento (juvenil e adulto), pela morfologia e aderência dos somitos abdominais no cefalotórax (selado nos juvenis e não selado nos adultos). Todos os siris foram dissecados, tendo seus estágios de desenvolvimento gonadal registrados pelo aspecto macroscópico das gônadas, atendo-se à sua coloração e tamanho em relação ao hepatopâncreas (PINHEIro \& Fransozo 1998).

A proporção de sexos (machos: fêmeas) foi calculada mensalmente e para o total de indivíduos estudados, com o contraste testado estatisticamente pelo teste de Qui-quadrado $\left(\chi^{2}\right)$.

Para análise da estrutura populacional, foram confeccionados histogramas mensais de distribuição de freqüência da largura cefalotorácica (LC), em classes de $0,5 \mathrm{~mm}$ de intervalo. Os dados foram empregados também para análise do recrutamento com o software FiSAT (FAO-ICLARM Stock Assessment Tools) (GAYANILlo et al. 1996), utilizando os parâmetros de crescimento obtidos por Branco \& Lunardon-Branco (1993b) para uma população de latitude similar.

O percentual de indivíduos adultos (gônadas em maturação + gônadas maturas), para cada classe de tamanho, foi empregado na confecção das curvas de maturidade sexual, tendo como variável dependente a freqüência de adultos, e independente, as classes de tamanho. As curvas de maturidade foram obtidas pelo ajuste da ogiva de Galton ( $\mathrm{y}=1$-e-A.Z, onde $\mathrm{Z}=\mathrm{xb}$ ) aos dados, cuja linearização é obtida por $\ln [\ln (1-\mathrm{y})]=$ $\ln A+b \cdot \ln x$. A estimativa do tamanho de primeira maturação fisiológica foi obtida por LC50\% = e $[\ln [-\ln (1-0,50)]-\ln A] / b$ (Fonteles-Filho 1989).

A relação entre o peso úmido e a largura da carapaça para cada sexo foi determinada por análise de regressão com ajuste da função potência. Posteriormente, foi determinado o nível de alometria para cada caso, com confirmação pelo teste $\mathrm{t}$, em isométrico $(\mathrm{b}=3)$, alométrico positivo $(\mathrm{b}>3)$ ou alométrico negativo $(\mathrm{b}<3)$ (HaRTNOLL 1982). Os indivíduos danificados ou com organismos epibiontes foram excluídos das análises.

\section{RESULTADOS}

De abril/2000 a abril/2001 foram coletados 1.671 indivíduos de C. ornatus, representados por 989 machos (505 juvenis e 484 adultos) e 682 fêmeas (517 juvenis, 162 adultas não ovígeras e 3 ovígeras). Todos os estágios de desenvolvimento, com exceção das fêmeas ovígeras, estiveram presentes em todos os meses de amostragem (Tab. I).

Os indivíduos juvenis foram dominantes em relação aos adultos para ambos os sexos, sendo mais abundantes no verão. Do total de fêmeas adultas obtidas durante as amostragens, apenas três eram ovígeras, tendo ocorrido duas em junho e uma em fevereiro/2001 (Tab. I).

A proporção de sexos total (machos:fêmeas) foi de 1,5:1, com uma variação de 0,1:1 em maio/2000 a 7,7:1 em outubro/2000 (Fig. 1). Em geral, os machos predominaram sobre as fêmeas $(1,5: 1,0)\left(\chi^{2}=124,2 ; \mathrm{GL}=12 ; \mathrm{p}<0,05\right)$. Durante o período verificou-se uma maior proporção de machos, exceto em maio, junho e março/2001, quando as fêmeas preponderaram, embora não tenha sido confirmado contraste em relação aos machos $(p>0,05)$ (Tab. I).

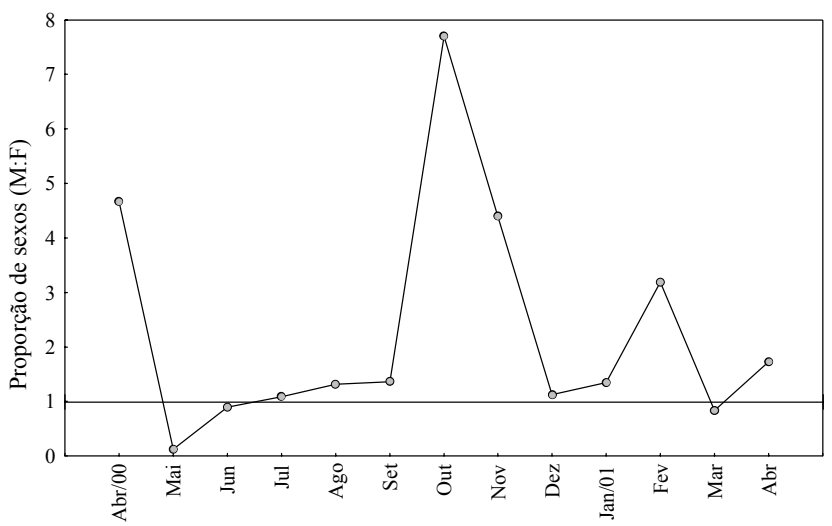

Figura 1. Variação da proporção entre machos (M) e fêmeas (F) de Callinectes ornatus ao longo do período de coletas no Balneário Shangri-lá, Paraná. Os pontos acima da unidade (linha) indicam predomínio de machos, enquanto aqueles abaixo, de fêmeas.

A distribuição mensal dos indivíduos em classes de tamanho (Fig. 2) indica dois períodos com elevada abundância nas classes de menor porte. Isto foi confirmado pela análise de recrutamento pelo FiSAT, que confirmou dois recrutamentos anuais com intensidade similar para $C$. ornatus, nos seguintes períodos: julho-agosto $(47,2 \%)$ e de novembro-dezembro $(52,8 \%)$.

Do total de 989 machos, 961 tiveram o grau de maturação gonadal determinado, estando 523 imaturos $(54,4 \%), 344$ em maturação $(35,8 \%)$ e 94 maturos $(9,8 \%)$. Indivíduos pertencentes a estes estágios gonadais estiveram sempre presentes em todos os meses analisados. Os machos imaturos apresentaram uma abundância constante de abril a novembro/2000, apresentando um pico expressivo em janeiro e outro menor em março/2001. Os machos em maturação apresentaram três pi- 

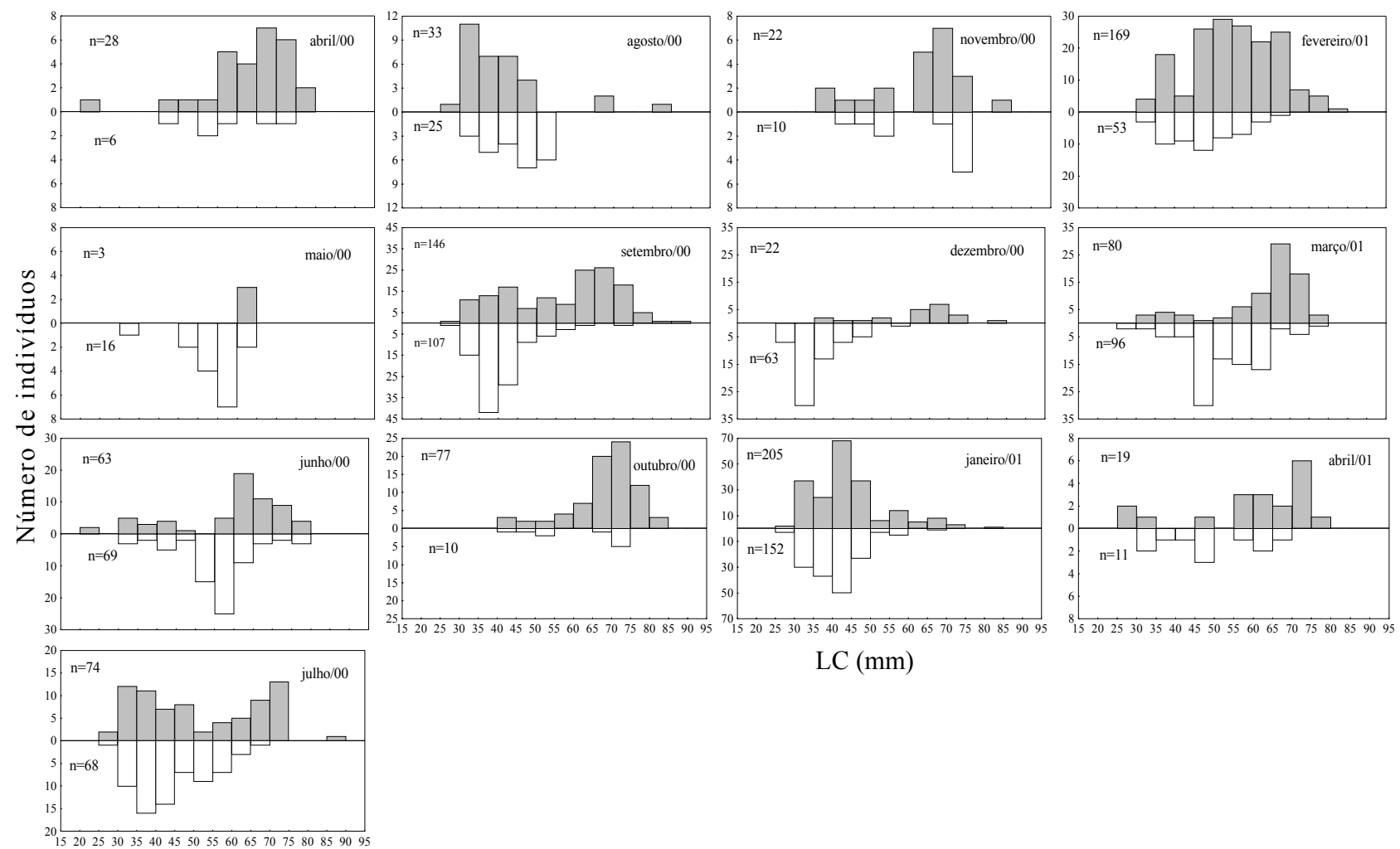

$\mathrm{LC}(\mathrm{mm})$

$\mathrm{LC}(\mathrm{mm})$

Figura 2. Distribuição de freqüência de largura da carapaça (LC) de Callinectes ornatus ao longo do período de coleta no Balneário Shangri-lá, Paraná. (barras cinzas) Machos, (barras brancas) fêmeas.

Tabela I. Freqüência de indivíduos de Callinectes ornatus em diferentes estágios de desenvolvimento e entre os sexos ao longo do período de coleta utilizando teste do Qui-quadrado $\left(\chi^{2}\right)$. ${ }^{*} p<0,05=$ diferença entre os sexos; (RS) razão sexual.

\begin{tabular}{|c|c|c|c|c|c|c|c|c|c|}
\hline \multirow{2}{*}{ Mês } & \multicolumn{4}{|c|}{ Fêmeas } & \multicolumn{3}{|c|}{ Machos } & \multirow{2}{*}{ RS (Macho:Fêmea) } & \multirow{2}{*}{$\chi^{2}$} \\
\hline & Jovem & Adulto & Ovígera & Total & Jovem & Adulto & Total & & \\
\hline Abril/2000 & 3 & 3 & - & 6 & 4 & 24 & 28 & $4,7: 1$ & $14,24^{*}$ \\
\hline Maio & 1 & 15 & - & 16 & 0 & 2 & 2 & $0,1: 1$ & $10,89^{*}$ \\
\hline Junho & 29 & 39 & 2 & 70 & 16 & 47 & 63 & $0,9: 1$ & 0,37 \\
\hline Julho & 46 & 22 & - & 68 & 40 & 34 & 74 & $1,1: 1$ & 0,25 \\
\hline Agosto & 19 & 6 & - & 25 & 30 & 3 & 33 & $1,3: 1$ & 1,10 \\
\hline Setembro & 97 & 10 & - & 107 & 55 & 91 & 146 & $1,4: 1$ & $6,01^{*}$ \\
\hline Outubro & 2 & 8 & - & 10 & 6 & 71 & 77 & $7,7: 1$ & 51,60 * \\
\hline Novembro & 0 & 5 & - & 5 & 6 & 16 & 22 & $4,4: 1$ & $10,70^{*}$ \\
\hline Dezembro & 63 & 0 & - & 63 & 61 & 10 & 71 & $1,1: 1$ & 0,48 \\
\hline Janeiro/2001 & 145 & 7 & - & 152 & 181 & 24 & 205 & $1,3: 1$ & $7,87^{*}$ \\
\hline Fevereiro & 46 & 6 & 1 & 53 & 82 & 87 & 169 & $3,2: 1$ & $60,61^{*}$ \\
\hline Março & 59 & 37 & - & 96 & 16 & 64 & 80 & $0,8: 1$ & 1,45 \\
\hline Abril & 7 & 4 & - & 11 & 8 & 11 & 19 & $1,7: 1$ & 2,13 \\
\hline Total & 517 & 162 & 3 & 682 & 505 & 484 & 989 & $1,5: 1$ & \\
\hline
\end{tabular}


cos de abundância (junho/2000, dezembro/2000 e março/2001). No período de abril a setembro/2000, os machos maturos apresentaram uma abundância constante, havendo um aumento em novembro-dezembro/2000 e um pico expressivo em março/2001, quando chegou a superar em abundância as demais categorias. Os machos imaturos mostraram uma maior representatividade percentual na maioria dos meses, ao contrário dos maturos que preponderaram em março/2001 (Fig. 3).

Do total de 682 fêmeas, 654 tiveram o grau de maturação gonadal determinado, das quais 438 estavam imaturas (67\%), 137 em maturação $(20,9 \%)$ e 79 maturas $(12,1 \%)$. Apenas os indivíduos em maturação estiveram presentes em todos os meses analisados. As fêmeas imaturas apresentaram dois picos de maior abundância (setembro/2000 e janeiro/2000), enquanto aquelas em maturação apresentaram certa constância durante o período amostral e as maturas ocorreram apenas de maio a julho/2000 e de fevereiro a março/2001. As fêmeas imaturas tiveram maior representatividade percentual na maioria dos meses, aquelas em maturação dominaram em abril, outubro e dezembro/2000 e as maturas em maio e junho/2000 (Fig. 3).

Foram utilizados 638 machos e 459 fêmeas para a análise da maturidade fisiológica. A curva de maturidade indica que $50 \%$ dos indivíduos estavam maturos com $55 \mathrm{~mm}$ (machos) e $48 \mathrm{~mm}$ (fêmeas) (Fig. 4). Os valores médios de largura da carapaça e peso para os machos foram, respectivamente, de $48,54 \mathrm{~mm}$ ( $\pm 14,10 \mathrm{~mm} \mathrm{DP})$ e $19,25 \mathrm{~g} \mathrm{(} \pm 14,88 \mathrm{~g} \mathrm{DP}) ;$ e $40,71 \mathrm{~mm}$ ( $\pm 10,32 \mathrm{~mm} \mathrm{DP}) 10,35 \mathrm{~g}( \pm 8,30 \mathrm{~g} \mathrm{DP})$ para as fêmeas.

As regressões entre o peso úmido $(\mathrm{P})$ e a largura da carapaça (LC) para cada sexo mostraram-se significativas, revelando que numa mesma classe de tamanho, os machos são mais pesados do que as fêmeas. As equações obtidas encontram-se na figura 5. A análise do nível de alometria demonstrou que o tipo de crescimento foi alométrico positivo tanto para machos $(\mathrm{t}=8,54 ; \mathrm{p}<0,05)$, quanto para fêmeas $(\mathrm{t}=3,74 ; \mathrm{p}<0,05)$.

\section{DISCUSSÃO}

A estrutura populacional dos crustáceos tem sido geralmente analisada pela distribuição dos exemplares em classes de tamanho, razão sexual, dinâmica temporal, crescimento e taxas de natalidade/mortalidade (NAKAGAKI \& PINHeIro 1999). O presente estudo revelou um predomínio de indivíduos jovens de $C$. ornatus, sendo rara a presença de fêmeas ovígeras. A ausência de alguns componentes da população, provavelmente devido ao comportamento migratório, impossibilitou o estudo do crescimento e o cálculo das taxas de natalidade e mortalidade.

A análise da distribuição de freqüência dos indivíduos nas classes de tamanho evidenciou dois recrutamentos anuais, um deles no inverno (julho-agosto) e outro na primavera (novembro-dezembro), apesar de existirem pulsos de recrutamento de menor intensidade ocorrendo em outros meses, que pode ser explicado pelas desovas múltiplas que caracterizam a maioria dos portunídeos (Pinheiro \& Fransozo 1999, 2002). Mantellato \& Fransozo (1998), em Ubatuba (São Paulo), verificaram uma desova contínua de $C$. ornatus com incidências mais elevadas de fêmeas ovígeras no outono. Em Matinhos (Paraná), BRANCo \& LUNARDON-BRANCO (1993a) observaram uma maior freqüência de fêmeas ovígeras na primavera e dos juvenis de agosto a novembro. Negreiros-Fransozo et al. (1999) observaram um padrão contínuo de recrutamento em Ubatuba, embora o regis- tro de maior número de juvenis tenha ocorrido de setembro a novembro. Neste sentido, percebe-se que o padrão observado encontra respaldo na literatura, valendo ressaltar que certas diferenças são esperadas em função da latitude, variações interanuais, ou até mesmo do petrecho de pesca utilizado.

Segundo Mantellato \& Fransozo (1998), C. ornatus apresenta um padrão reprodutivo contínuo, com maior atividade durante o verão e o outono, quando a presença de machos e fêmeas com gônadas maduras e fêmeas ovígeras é mais evidente. O mesmo foi observado por BRANCo \& LunARDon-Branco (1993a), embora o estudo destes autores não tenha registrado fêmeas ovígeras.

No presente estudo, as fêmeas ovígeras também não foram bem representadas, tendo sido coletadas apenas duas em junho e uma em fevereiro, no entanto o estudo da maturação gonadal mostrou que a maior freqüência de fêmeas maduras ocorreu do final do outono ao início do inverno (maio a julho), diferindo, portanto, da época de reprodução observada nos trabalhos de Branco \& Lunardon-Branco (1993a) e Mantellato \& Fransozo (1998).

O tamanho de primeira maturação para C. ornatus foi de $55 \mathrm{~mm}$ para os machos e $48 \mathrm{~mm}$ para as fêmeas. BRANCO \& LUNARDON-BRANCO (1993b) evidenciaram a maturação desta espécie com um maior porte (67 e $61 \mathrm{~mm}$, respectivamente), enquanto para Mantellato \& Fransozo (1996) os valores obtidos foram de $50 \mathrm{~mm}$ (machos) e $43 \mathrm{~mm}$ (fêmeas), bem próximos daqueles obtidos no presente estudo. Essas pequenas variações intraespecíficas no tamanho de primeira maturação podem ocorrer em função da origem da população, bem como outros fatores abióticos envolvidos no processo. O início da maturidade sexual varia, consideravelmente, entre as espécies de siris, entre populações da mesma espécie e até entre indivíduos da mesma população, estando geralmente relacionado com o alcance de um determinado tamanho individual (Fonteles-Filho 1989). No presente estudo, os machos apresentaram tamanho na maturidade maior do que as fêmeas, indicando um dimorfismo sexual em relação a este parâmetro. Esta característica representa uma importante adaptação para a cópula, pois machos maiores têm maior facilidade na manipulação das fêmeas durante a cópula, além de maximizar o potencial de combate com outros machos pela parceira.

A relação entre o peso e o tamanho pode fornecer informações a respeito do crescimento, maturação gonadal, dimorfismo sexual e proximidade da muda. Comparando os valores médios da largura da carapaça e do peso, assim como as retas obtidas para machos e fêmeas, observa-se que os machos apresentaram um crescimento um pouco mais expressivo do que as fêmeas. Branco \& Lunardon-Branco (1993a) também observaram que os machos possuem um peso superior ao das fêmeas de uma mesma classe de largura da carapaça. Após a muda da puberdade, quando os indivíduos já estão aptos fisiológica e morfologicamente para a reprodução, os sexos usualmente apresentam diferentes taxas de crescimento (HARTNOLL 1985). Essas diferenças alométricas entre os sexos podem ser atribuídas ao maior gasto energético das fêmeas para a gametogênese do que os machos, que por sua vez podem investir mais energia ao crescimento somático (Mantellato \& MarTinelli 1999).

No presente estudo, C. ornatus apresentou um crescimento alométrico positivo independente do sexo considerado, corroborando os dados de Mantellato \& Martinelli (1999) em 


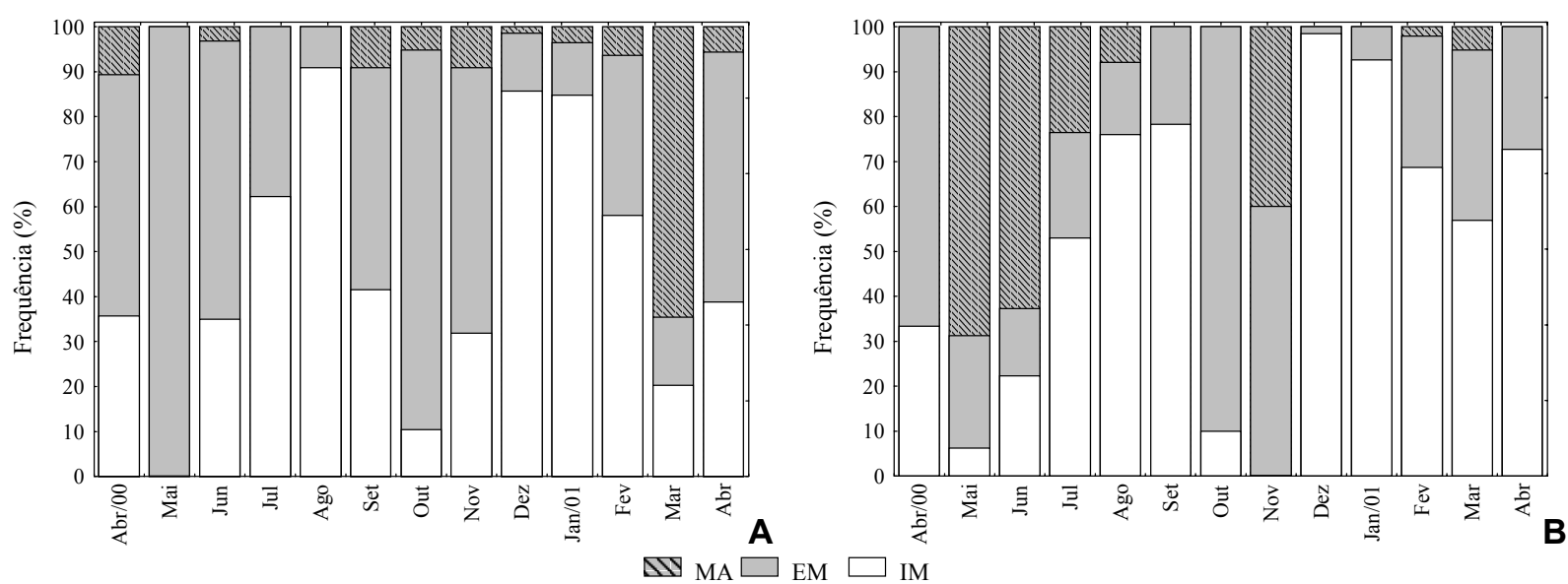

Figura 3. Freqüência relativa (\%) dos estágios de maturação dos machos (A) e das fêmeas (B) de Callinectes ornatus, no Balneário Shangri-lá, Paraná, ao longo do período de coleta. (IM) Imaturo, (EM) em maturação, (MA)maduro.
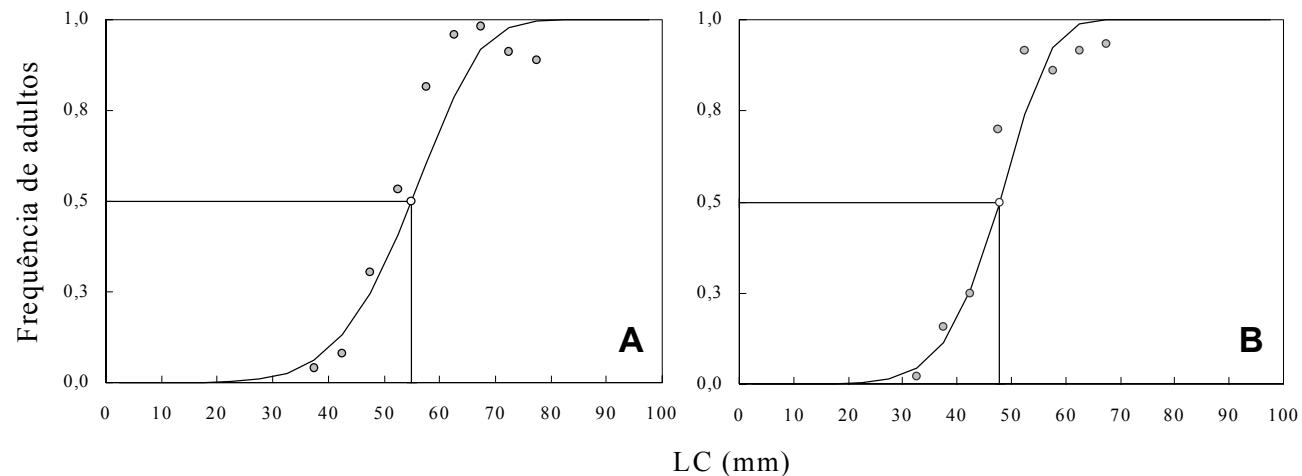

Figura 4. Curva de maturidade fisiológica dos machos (A) e das fêmeas (B) de Callinectes ornatus no Balneário Shangri-lá, Paraná, durante o período de coletas, indicando a classe onde $50 \%$ dos indivíduos eram adultos.
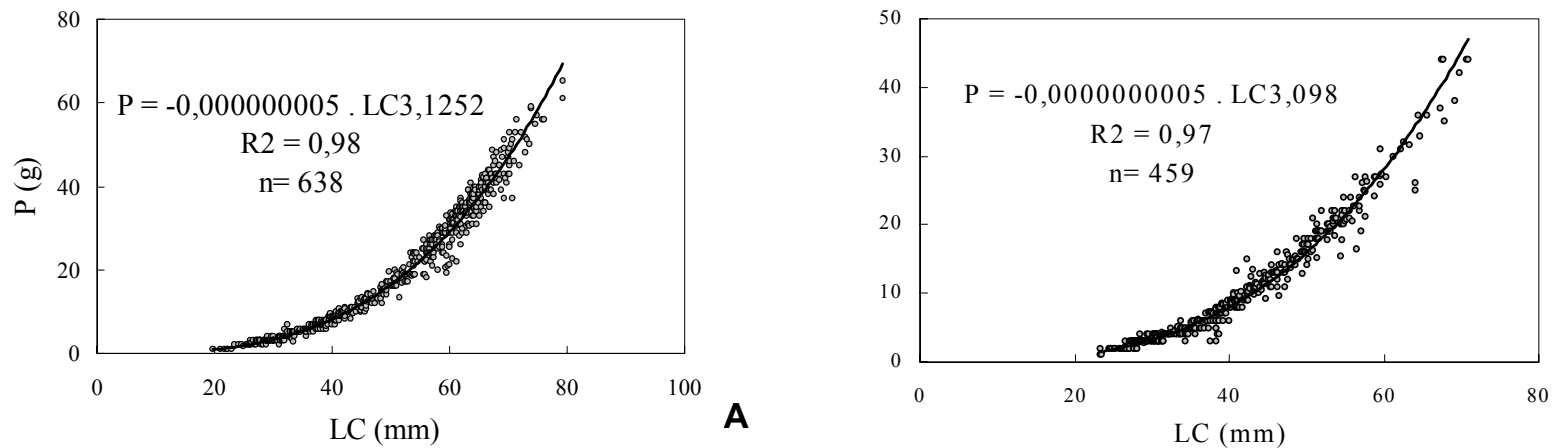

Figura 5. Regressão entre o peso úmido (P) e a largura da carapaça (LC) dos machos (A) e das fêmeas (B) de Callinectes ornatus no Balneário Shangri-lá, Paraná, durante o período de coletas.

Ubatuba. Apesar dos métodos de mensuração utilizados por estes autores serem diferentes do presente estudo, impedindo a comparação das equações, pode-se inferir que ocorram vari- ações do padrão de crescimento em função da época e local estudado, devendo-se, principalmente, à heterogeneidade ambiental (PinHeiro et al. 1993). 
Callinectes ornatus possui um ciclo de vida complexo, existindo fases características da região estuarina e outras de mar aberto, além de diferenças na distribuição das classes etárias e na proporção de sexos, conforme observado por PITA et al. (1985) na Baía de Santos (São Paulo). Estes autores registraram animais de menor porte no interior da Baía, com as fêmeas se concentrando em águas mais rasas quando juvenis.

A existência de grandes estuários na região (e.g. Baía de Guaratuba e Baía de Paranaguá), sugere que estudos de dinâmica populacional e da biologia reprodutiva de $C$. ornatus devem prever amostragens dentro e fora do estuário, a partir de um gradiente de profundidade e salinidade, favorecendo a captura representativa de todas as parcelas da população.

\section{AGRADECIMENTOS}

Ao Curso de Pós-Graduação em Zoologia da Universidade Federal do Paraná e ao Centro de Estudos do Mar (CEM) pelo apoio durante todas as fases dessa pesquisa. A CAPES pela bolsa de mestrado concedida para primeira autora. A Cooperativa de pescadores de Shangri-lá que possibilitaram a realização das coletas. Ao Biólogo MSc. Rafael Metri e a Prof. Dra. Setuko Masunari pela leitura crítica e sugestões desse manuscrito.

\section{REFERÊNCIAS BIBLIOGRÁFICAS}

Branco, J.O. \& M.J. Lunardon-Branco. 1993a. Aspectos da biologia de Callinectes ornatus Ordway, 1863 (Decapoda, Portunidae) da região de Matinhos, Paraná, Brasil. Arquivos de Biologia e Tecnologia, Curitiba, 36 (3): 489-496.

- 1993b. Crescimento e tamanho de primeira maturação em Callinectes ornatus Ordway, 1863 (Decapoda, Portunidae) da região de Matinhos, Paraná, Brasil. Arquivos de Biologia e Tecnologia, Curitiba, 36 (3): 497-503.

FAO (Food and Agriculture Organization of the United Nations). 1993. Guia de campo de las especies comerciales marinas y de aguas salobras de la costa septentrional de Sur America. Rome, FAO, Comisión de Comunidades Europeas y de NORAD, 149p.

Fonteles-Filho, A.A. 1989. Recursos pesqueiros. Biologia e dinâmica populacional. Fortaleza, Imprensa Oficial do Ceará, 296p.

Gayanillo Jr., F.C.; P. Sparre \& P. Pauly. 1996. FAO-IClARM Stock Assessment Tools - User's manual. Rome, FAO, 126p.

HaEfner JR., P.A. 1990. Morphometry and size at maturity of Callinectes ornatus (Brachyura: Portunidae) in Bermuda. Bulletin of Marine Science, Miami, 46 (2): 274-286.

Hartnoll, R.G. 1982. Growth, p. 11-196. In: B.E. Bliss \& L.G. AвELE (Eds). The biology of Crustacea: Embriology, Morphology and Genetics. New York, Academic Press, 402p.

- 1985. Growth, sexual maturity and reproductive output, p. 101-128. In: A. WENNER (Ed.). Crustacean Issues, 3: factors in adult growth. The Netherlands, Balkema Publishers, 375p.

JoRDAN, S.J. 1998. The blue crab fisheries of North America: research, conservation, and management. Journal of Shellfish Research, New York, 17 (2): 1-577.

Lipcius, R.N. \& W.A. VAN ENGel. 1990. Blue crab population dynamics in Chesapeake Bay: variation in abundance (York River, 1972-1988) and stock-recruit functions. Bulletin of Marine Science, Miami, 46 (1): 180-194.

Mantellato, F.L.M \& A. Fransozo. 1996. Size at sexual maturity in Callinectes ornatus (Brachyura, Portunidae) from the Ubatuba region (SP), Brazil. Nauplius, Rio Grande, 4: 29-38. . 1997. Fecundity of the crab Callinectes ornatus Ordway, 1863 (Decapoda, Brachyura, Portunidae) from the Ubatuba region, São Paulo, Brazil. Crustaceana, Leiden, 70 (2): 214-226.

. 1998. Reproductive biology and moulting cycle of the crab Callinectes ornatus Ordway, 1863 (Decapoda, Brachyura, Portunidae) from the Ubatuba region, São Paulo, Brazil. Crustaceana, Leiden, 72 (1): 63-76.

Mantellato, F.L.M \& J.M. Martinelli. 1999. Carapace widthweight relationships of Callinectes ornatus Ordway, 1863 (Brachyura, Portunidae) from the Ubatuba Bay, Brazil. Iheringia, Série Zoologia, Porto Alegre, 87: 111-116.

Melo, G.A.S. 1996. Manual de Identificação dos Brachyura (caranguejos e siris) do litoral brasileiro. São Paulo, Ed. Plêiade/FAPESP, 604p.

. 1999. Infraordem Brachyura. Siris e caranguejos: espécies marinhas e estuarinas, p. 415-485. In: L. BucKuP \& G. Bond-Buckup (Eds.). Os crustáceos do Rio Grande do Sul. Porto Alegre, Ed. da Universidade Federal do Rio Grande do Sul, 503p.

NaKagaKi, J.M. \& M.A. Pinheiro. 1999. Biologia populacional de Emerita brasiliensis Schmitt (Crustacea, Hippidae) na Praia Vermelha do Norte, Ubatuba (São Paulo, Brazil). Revista Brasileira de Zoologia, Curitiba, 16 (2): 83-90.

Negreiros-Fransozo, M.L; F.LM. Mantelatto. \& A. Fransozo. 1999. Population biology of Callinectes ornatus Ordway, 1863 (Decapoda, Portunidae) from Ubatuba (SP), Brazil. Scientia Marina, Barcelona, 63 (2): 157-163.

Pinheiro, M.A.A. \& A. Fransozo. 1998. Sexual maturity of speckled swimming crab Arenaeus cribrarius (Lamark, 1818) (Crustacea, Brachyura, Portunidae) in the Ubatuba Littoral, São Paulo State, Brazil. Crustaceana, Leiden, 71 (4): 434-452.

. 1999. Reproductive behavior of the swimming crab Arenaeus cribrarius (Lamark, 1818) (Crustacea, Brachyura, Portunidae) in captivity. Bulletin of Marine Science, Mia$\mathrm{mi}, 64$ (2): 243-253.

. 2002. Reproduction of speckled swimming crab Arenaeus cribrarius (Lamark, 1818) (Brachyura, Portunidae) on the Brazilian coast near $23^{\circ} 30^{\prime} \mathrm{S}$. The Journal of Crustacean Biology, Lawrence, 22 (2): 416-428.

Pinheiro, M.A.A.; A. Fransozo \& M.L. Negreiros-Fransozo. 1993. Relative growth of the speckled swimming crab Arenaeus cribarius (Lamarck, 1818) (Crustacea, Portunidae). Arquivos de Biologia Tecnologia, Curitiba, 36 (2): 331-341.

Pita, J.B.; E.S. Rodrigues; R. Graça-Lopes \& J.A.P. Coelho. 1985. Levantamento preliminar da Família Portunidae (Crustacea, Decapoda, Brachyura) no Complexo Baía-estuário de Santos, São Paulo, Brasil. Boletim do Instituto de Pesca, São Paulo, 12 (3): 153-162.

WilLiams, A.B. 1974. The swimming crabs of the genus Callinectes (Decapoda: Portunidae). Fishery Bulletin, Seattle, 72 (3): 685-798.

. 1984. Shrimps, lobsters end crabs of the Atlantic coast of the Eastern United States: Maine to Florida. Washington, Smithsonian Institution Press, 549p.

Recebido em 17.VI.2003; aceito em 30.X.2003. 